\title{
Eficácia do adesivo tecidual de fibrina na fixação de enxerto conjuntival autógeno em cirurgias de pterígio primário
}

\author{
Efficacy of fibrin tissue adhesive in the attachment of autogenous conjuntival graft \\ on primary pterygium surgery
}

Michel Risnic Rubin ${ }^{1}$, Paulo Elias C. Dantas ${ }^{1}$, M. Cristina Nishimaki-Dantas 2 , Sergio Felberg ${ }^{3}$

\section{RESUMO}

Objetivo: Comparar a eficácia da fixação do enxerto autógeno de conjuntiva ao leito escleral após exérese de pterígio primário, utilizando-se adesivo tecidual de fibrina ou sutura de monofilamento de nylon 10-0.

Métodos: Estudo comparativo, prospectivo e aleatório foi realizado em 47 olhos de 47 pacientes com pterígio primário de localização medial. O grupo 1 (adesivo) foi constituído de 21 pacientes submetidos à fixação do enxerto conjuntival com adesivo tecidual de fibrina (Quixil TM); enquanto que o grupo 2 (sutura), foi constituído de 26 pacientes, cuja fixação do enxerto foi feita com sutura em pontos separados de monofilamento de nylon 10-0 (Ethicon ${ }^{\circledR}$ ) após a excisão do pterígio feita pelo mesmo cirurgião. Os pacientes foram avaliados antes da cirurgia, no 1ำ, 14ํㅜ e $21^{\circ}$ dia após a cirurgia. Utilizou-se uma escala visual de desconforto ocular e verificou-se o tempo cirúrgico, hiperemia ocular e presença de complicações, além de sinais de recidiva, sendo sua presença também avaliada no $6^{\circ}$ mês após a cirurgia. As variáveis foram submetidas à análise estatística. Valores de $p<0,05$ indicaram significância estatística. Resultados: $\bigcirc$ tempo cirúrgico médio foi de 19,05 $\pm 6,12$ minutos para o grupo 1 e $48,15 \pm 7,13$ minutos para o grupo sutura $(p<0,001)$. A escala referente ao desconforto ocular demonstrou um menor escore no $1^{\circ}(p<0,005)$, $7^{\circ}(p<0,001)$ e $21^{\circ}$ $(p<0,001)$ dia após a cirurgia com a utilização do adesivo tecidual. A hiperemia ocular mostrou-se menor em todos os períodos do estudo após a cirurgia $(p<0,001)$ no grupo cola. As complicações encontradas, uma em cada grupo, resolveram-se com tratamento clínico até o $21^{\circ}$ dia após a cirurgia. Houve uma recidiva no grupo 1 e duas no grupo 2 até o $6^{\circ}$ mês após a cirurgia.

Conclusão: Em cirurgia de fixação de enxerto autógeno de conjuntiva para tratamento de pterígio primário, adesivo tecidual de fibrina reduziu o tempo do ato cirúrgico, desencadeou menor hiperemia conjuntival e desconforto no período pósoperatório, com índice de recidiva semelhante em comparação com o uso de sutura de mononylon 10.0, demonstrando ser uma excelente opção para a fixação do enxerto conjuntival no tratamento cirúrgico do pterígio primário.

Descritores: Túnica conjuntiva/transplante; Pterígio/cirurgia; Adesivo tecidual de fibrina/uso terapêutico; Suturas; Doenças da túnica conjuntiva/patologia; Doença enxerto-hospedeiro; Estudo comparativo

\begin{abstract}
Purpose: To compare the efficacy of conjunctival autograft surgery with the attachment to the scleral bed using fibrin tissue adhesive or mononylon 10-0 suture after resection of primary pterygium.

Methods: A comparative, prospective and randomized clinical trial was performed in 47 eyes of 47 patients with primary medial located pterygium. Group 1 (adhesive) was composed by 21 patients that underwent conjunctival autograft closure with fibrin tissue adhesive (Quixil'M) and Group 2 (suture) was composed by 26 patients that underwent pterygium surgery with mononylon 10-0 (Ethicon ${ }^{\circledR}$ ) suture (suture group) after pterygium excision. All surgeries were performed by the same surgeon. Patients were assessed on the preoperative period and on the $11^{\text {st }}, 14^{\text {th }}$ and $21^{\text {st }}$ postoperative days. They were followed-up with a questionnaire of ocular discomfort and by the surgical time spent, ocular hyperemia, complications and recurrence signals, being the recurrence also evaluated at the 6 postoperative month. Data were submitted to statistical analysis. A value of $p<0.005$ was considered statistically significant.

Results: The average surgical time was $19.05 \pm 6.12$ minutes in group 1 (glue) and 48.15 \pm 7.13 minutes in the group 2 (suture) $(p<0.001$ ). The ocular discomfort scale analysis showed a lower score in the $1^{\text {st }}(p<0.005), 7^{\text {th }}(p<0.001)$ and $21^{\text {th }}(p<0.001)$ postoperative days in group 1. Ocular hyperemia was less intense in all periods of this study in group 1 $(p<0.001)$. Complications were one in each group and both were managed with clinical treatment until the $21^{\text {th }}$ postoperative day. There was one recurrence in group 1 and two in group 2 until the $6^{\text {th }}$ postoperative month.

Conclusion: In the surgical management of primary pterygium, fibrin tissue adhesive attached the conjunctival autograft, decreased the surgical time and diminished the conjunctival hyperemia and ocular discomfort with similar recurrences on the postoperative period, compared to fixation with mononylon 10.0 suture, proving to be an excellent option for conjunctival autograft attachment in primary pterygium surgery.
\end{abstract}

Keywords: Conjunctiva/transplantation; Pterygium/surgery; Fibrin tissue adhesive/therapeuticuse; Sutures; Conjunctival diseases/pathology; Graftvs host disease; Comparative study

\section{INTRODUÇÃO}

Pterígio é caracterizado por proliferação fibrovascular anômala proveniente da conjuntiva bulbar sobre o limbo e a córnea, com etiopatogenia incerta ${ }^{(1-4)}$. Acredita-se que seja causado e agravado por aumento da exposição à radiação ultravioleta, micro-traumatismos e inflamações crônicas ${ }^{(4-6)}$. Sua incidência pode variar de 4 a $11 \%$ em climas tropicais ${ }^{(3-5,7-8)}$
Cobertura da área desnuda por enxerto conjuntival vem sendo amplamente utilizada no manejo cirúrgico do pterígio, devido ao baixo porcentual de recidivas em pterígios primários e recidivados, variando na literatura em torno de $5 \%$ em todos os estádios de classificações pré-operatórias ${ }^{(9-11)}$. A principal opção atual de fixação do enxerto conjuntival é a sutura com fios absorvíveis e inabsorvíveis. Este tipo de fixação apresenta o inconveniente de demandar
Submitted for publication: October 27, 2009

Accepted for publication: May 9, 201

Study carried out at the Departamento de Oftalmologia da Irmandade da Santa Casa de Misericórdia de São Paulo - ISCMSP - São Paulo (SP) - Brasil.

Physician, Departamento de Oftalmologia, Irmandade da Santa Casa de Misericórdia de São Paulo ISCMSP - São Paulo (SP), Brasil.

2 Physician, Seção de Córnea e Doenças Externas, Diretora do Departamento de Oftalmologia, Physician, Seção de Cornea e Doenças Externas, Diretora do Departamento de Oftalmo
Irmandade da Santa Casa de Misericórdia de São Paulo - ISCMSP - São Paulo (SP), Brasil.

${ }^{3}$ Physician, Seção de Córnea e Doenças Externas, Departamento de Oftalmologia, Irmandade da Santa Casa de Misericórdia de São Paulo - ISCMSP - São Paulo (SP), Brasil.
Funding: No specific financial support was available for this study.

Disclosure of potential conflicts of interest: M.R.Rubin, None; P.E.C.Dantas, None; M.C.NishiwakiDantas, None; S. Felberg, None.

Correspondence address: Michel Risnic Rubin. Rua Martin Francisco, 67 - Apto. 63 - São Paulo SP - 01226-001 - Brazil - E-mail: rubinmichel@hotmail.com

Editorial Note: After completing the confidential analysis of the manuscript, ABO discloses, with his agreement, the name Dr. Samuel Rymer as a reviewer. We thank his effort and expertise in participating in this process 
tempo cirúrgico relativamente longo, promover maior reação inflamatória, causar incômoda sensação de corpo estranho no período pós-operatório e desconforto provocado pela retirada dos pontos ${ }^{(5)}$.

Recentemente, foi desenvolvida uma técnica de fixação do enxerto conjuntival utilizando-se adesivo tecidual de fibrina, com diminuição do tempo cirúrgico e do desconforto após a cirurgia, além de não produzir a sensação de corpo estranho causada pelos fios de sutura e não ser necessária sua retirada ${ }^{(12-13)}$

$\mathrm{O}$ adesivo tecidual de fibrina Quixil ${ }^{\mathrm{TM}}$ (Johnson e Johnson's, Rehovot, Israel) é formada por dois componentes derivados do plasma humano proveniente de doadores, sendo uma solução de concentrado de proteínas plasmáticas $(40-60 \mathrm{mg} / \mathrm{ml})$ que contém fibrinogênio, fibronectina, fator XIII e fator de von Wilebrand e uma solução de trombina (900-1100 IU/mL) contendo cálcio. Ao serem aplicadas à superfície tecidual, as duas soluções misturam-se e após aproximadamente 30 segundos, reproduzem os estádios finais da cascata de coagulação, formando fina camada de fibrina, sendo esta absorvida em poucos dias e, então, substituída por colágeno cicatricial ${ }^{(1,12-14)}$.

O objetivo deste estudo foi comparar a eficácia da fixação do enxerto de conjuntiva ao leito escleral utilizando-se adesivo tecidual de fibrina com a sutura de Mononylon ${ }^{\circledR}$ após exérese de pterígio primário.

\section{MÉTODOS}

Estudo prospectivo, controlado e aleatório foi desenvolvido com dois grupos, constituídos por 47 pacientes que procuraram espontaneamente nosso hospital. O presente estudo foi aprovado pelo comitê de ética em pesquisa da Irmandade da Santa Casa de Misericórdia de São Paulo.

Seguiram-se os critérios de inclusão e exclusão descritos abaixo:

Critérios de Inclusão

- Diagnóstico de pterígio medial primário, com queixas a ele relacionadas, cujo portador desejasse realizar o procedimento cirúrgico.

- Idade entre 18 a 70 anos.

- Concordar com o termo de ciência e consentimento.

Critérios de exclusão

- Diagnóstico suspeito ou confirmado de glaucoma.

- Doença corneal ou da superfície ocular associada.

- Mal estado geral, ASA 3 e 4.

Os pterígios foram graduados de acordo com sua extensão em relação ao limbo em

- Grau l: até $2 \mathrm{~mm}$

- Grau II: estendendo-se de 2 a $4 \mathrm{~mm}$.

- Grau III: estendendo-se acima de $4 \mathrm{~mm}$.

Um mesmo cirurgião treinado e habilitado realizou todos os procedimentos cirúrgicos. Um observador independente e experiente realizou as avaliações pré e pós-operatórias (PO).

Após anestesia tópica com colírio anestésico de tetracaína a 1\% e infiltração do corpo do pterígio com lidocaína a 2\%, a aderência da cabeça do pterígio à córnea foi desfeita com lâmina fria. Realizada dissecção e exérese após o limbo com tesoura de conjuntiva, seguida pela excisão da cápsula de Tenon. Foi evitada cauterização do sangramento. Enxerto de conjuntiva superior ipsilateral foi marcado horizontalmente e verticalmente, com $2 \mathrm{~mm}$ a mais que as medidas da conjuntiva excisada; este foi movido para área medial do limbo esclerocorneal e fixado à esclera, pelos modos descritos a seguir:

- Grupo 1 (Adesivo tecidual de fibrina): Foram operados 21 olhos. Aplicada 1 gota da cola de fibrina Quixil ${ }^{T M}$ na base da esclera, seguida da acomodação do enxerto em até 30 segundos, após este ter sido cuidadosamente deslocado ao local desejado. Eventual excesso de cola era removido.

- Grupo 2 (Sutura): Foram operados 26 olhos. Realizados oito pontos simples de sutura de mononylon 10-0 (Ethicon $\left.{ }^{\circledR}\right)$, separados e equidistantes.

A escolha da forma de fixação foi aleatória. A orientação apropriada foi mantida deixando-se sempre o epitélio exposto e a superfície límbica do enxerto ficando em contato com o limbo corneal.

Após a cirurgia, iniciou-se o uso de cloridrato de ciprofloxacina $3,5 \mathrm{mg} / \mathrm{ml}$ associado a dexametasona $1 \mathrm{mg} / \mathrm{ml}$ (Biamotil D, Allergan ${ }^{\circledR}$ ), 1 gota de $6 / 6$ horas, até o $21^{\circ}$ dia PO.

Nos dias 1, 7 e 21 após a cirurgia, os pacientes foram avaliados, com aferição da acuidade visual, biomicroscopia à lâmpada de fenda, graduação da hiperemia conjuntival, medida da pressão intraocular e marcação da Escala Análoga Visual (EAV). No 21ํ dia PO, todas as suturas deste grupo foram removidas. Ainda no $6^{\circ}$ mês PO os pacientes foram reavaliados quanto a recidivas.

EAV consistia na marcação pelo paciente de escore variável entre 0 e 10, no qual 0 representava ausência de desconforto ocular e 10 o pior desconforto ocular possível.

A hiperemia conjuntival foi graduada por um mesmo observador independente em: grau 0, ausente (vasos normais); I, leve (discreta injeção vascular); II, moderada (hiperemia ocular difusa); III, intenso (hiperemia ocular difusa com intensa dilatação dos vasos conjuntivais).

Foram verificados eventuais sinais de complicações ou recidivas, definidas como invasão tecidual ultrapassando o limbo corneal.

As variáveis receberam análise estatística, utilizando-se o método t de Student e o teste não-paramétrico de Mann-Whitney para avaliação das análises quantitativas. Para a comparação dos momentos de avaliação dentro de um mesmo grupo, foi considerado o teste nãoparamétrico de Friedman. Em relação às variáveis dicotômicas, os grupos foram comparados usando-se o teste Exato de Fisher. Valores de $p<0,05$ eram considerados estatisticamente significantes.

\section{RESULTADOS}

O tempo médio de cirurgia foi de 19,05 $\pm 6,12$ minutos no grupo 1 (adesivo) e 48,5 \pm 7,13 minutos no grupo 2 (sutura) com $p<0,001$ (Tabela 1).

A análise da escala análoga visual (EAV) demonstrou escore semelhante no período pré-operatório $(p=0,367)$ e escore significantemente menor no grupo 1 em todos os períodos pós-operatórios (PO) em estudo (Tabela 2). Verificou-se, ainda, que a EAV no 1ํ dia $\mathrm{PO}$ do grupo 1 foi semelhante ao $7^{\circ}(p=0,438)$ e $21^{\circ} \mathrm{PO}(p=0,205)$ do grupo 2

A hiperemia ocular no grupo 1 foi menor em todo período pósoperatório estudado (Tabela 3, Figura 1). Verificou-se que também a hiperemia ocular no grupo 1 no $1^{\circ}$ dia $\mathrm{PO}$ foi semelhante ao $7^{\circ}$ $(p=0,205)$ e ao dia $21^{\circ} \mathrm{PO}$ no grupo $2(p=0,477)$.

Não houve perda do enxerto em ambos os grupos. As complicações verificadas aconteceram no $7^{\circ}$ dia após a cirurgia, sendo um

Tabela 1. Tempo cirúrgico em minutos dos grupos 1 (adesivo) e 2 (sutura)

\begin{tabular}{cccccccc}
\hline Grupo & N (47 olhos) & Média & Mediana & Mínimo & Máximo & Desvio padrão & Valor de p \\
\hline 1 & 21 & 19,05 & 20,0 & 12 & 30 & 6,12 & $<0,001$ \\
2 & 26 & 48,15 & 48,5 & 35 & 60 & 7,13 & \\
\hline
\end{tabular}

* Teste t de Student para amostras independentes, $p<0,05$

Fonte: Ambulatório de Córnea e Doenças Externas do Departamento de Oftalmologia da Santa Casa de São Paulo. 
granuloma de conjuntiva no grupo 2, que se resolveu com a instilação de colírio de corticóide tópico até o $21^{\circ}$ dia PO, e uma retração do enxerto no grupo 1, com posterior conjuntivalização até o $21^{\circ}$ dia $\mathrm{PO}$, não sendo encontradas diferenças entre o número de complicações entre os grupos estudados $(p=1)$.

No 6ำ mês após a cirurgia, perdeu-se seguimento de 16 pacientes (34\%), sendo sete do grupo 1 e nove do grupo 2. Encontrou-se uma recidiva no grupo 1 e duas recidivas no grupo 2 , sendo detectadas: uma em ambos os grupos no $21^{\circ}$ dia após a cirurgia e uma no grupo 2 no $6^{\circ}$ mês após a mesma. Portanto quando comparada a toda amostra inicial, houve $4,76 \%$ de recidiva no grupo 1 e $7,69 \%$

Tabela 2 . Análise do escore relacionado ao desconforto ocular, Escala Análoga Visual (EAV), nos diferentes períodos estudados

\begin{tabular}{|c|c|c|c|c|c|}
\hline Período & Grupo & Média & Mediana & Desvio padrão & Valor de $p$ \\
\hline \multirow[t]{2}{*}{ Pré-op } & 1 & 5,10 & 5,0 & 1,26 & 0,367 \\
\hline & 2 & 4,77 & 4,5 & 1,21 & \\
\hline \multirow[t]{2}{*}{$1 \stackrel{\circ O}{P O}$} & 1 & 3,86 & 4,0 & 1,15 & 0,005 \\
\hline & 2 & 4,85 & 5,0 & 1,08 & \\
\hline \multirow[t]{2}{*}{$7^{\circ} \mathrm{PO}$} & 1 & 2,43 & 2,0 & 1,08 & $<0,001$ \\
\hline & 2 & 4,12 & 4,0 & 1,14 & \\
\hline \multirow[t]{2}{*}{$21^{\circ} \mathrm{PO}$} & 1 & 1,57 & 2,0 & 0,60 & $<0,001$ \\
\hline & 2 & 3,38 & 3,0 & 0,90 & \\
\hline
\end{tabular}

Pré-op= pré-operatório; $\mathrm{PO}=$ pós-operatório

*Teste não-paramétrico de Mann-Whitney, $p<0,05$

Fonte: Ambulatório de Córnea e Doenças Externas do Departamento de Oftalmologia da Santa Casa de São Paulo.

Tabela 3. Graduação da hiperemia ocular nos diferentes períodos em estudo

\begin{tabular}{lccccc}
\hline Período & Grupo & Média & Mediana & Desvio padrão & Valor de p \\
\hline 1 으 & 1 & 1,86 & 2 & 0,57 & $<0,001$ \\
$7 ㅇ ㅡ$ & 2 & 2,62 & 3 & 0,70 & \\
& 1 & 1,19 & 1 & 0,81 & $<0,001$ \\
$21 \mathrm{PO}$ & 2 & 2,12 & 2 & 0,65 & \\
& 1 & 0,71 & 1 & 0,72 & $<0,001$ \\
& 2 & 1,69 & 2 & 0,62 & \\
\hline
\end{tabular}

$\mathrm{PO}=$ pós-operatório

*Teste não-paramétrico de Mann-Whitney, $p<0,05$

Fonte: Ambulatório de Córnea e Doenças Externas do Departamento de Oftalmologia da Santa Casa de São Paulo. no grupo 2. Considerando apenas os pacientes que mantiveram seguimento até o $6^{\circ}$ mês após a cirurgia, tivemos $7,10 \%$ de recidiva no grupo 1 e 11,80\% no grupo 2, sem diferença significativa entre os grupos em ambas as situações $(p=1)$.

\section{DISCUSSÃO}

O uso da conjuntiva autógena ipsilateral, como fonte de enxerto conjuntival, está associada a risco mínimo de complicações, com baixa morbidade, além de baixo número de recidivas ${ }^{(10,15)}$. Optamos pela escolha, para o grupo controle, utilização do monofilamento de nylon 10.0 para fixação do enxerto conjuntival. A opção pelo fio monofilamentar inabsorvível foi devida às propriedades em causar menor reação inflamatória, tender a lesar menos os tecidos e provocar menos infecção, em comparação com os fios multifilamentares absorvível trançados, como a poliglactina (Vycril)(16).

$\mathrm{O}$ adesivo tecidual de fibrina Quixil ${ }^{\top M}$ é a única cola tecidua comercialmente disponível completamente isenta de componentes animais em sua composição(13). As colas de fibrina possuem risco de transmissão viral menor que outros hemoderivados, embora nenhuma transmissão viral tenha sido detectada com sua utilização, mesmo em maior volume, como as utilizadas em cirurgias hepáticas e ortopédicas. Os pacientes devem ser informados desse potencial risco antes da cirurgia(1)

Não há consenso sobre a cirurgia ideal. Acredita-se que os parâmetros mais eficazes para comparações sejam as recorrências, a resposta inflamatória e a presença de complicações ${ }^{(11)}$, aspectos estes utilizados neste estudo.

O menor tempo cirúrgico no grupo cola (grupo 1), deveu-se principalmente ao fato da não necessidade de sutura, sendo aproximadamente 2,5 vezes menor em comparação com a sutura (grupo 2). Outros estudos utilizando os diversos tipos de cola de fibrina existentes também demonstraram essa importante vantagem, com tempo cirúrgico significantemente menor em comparação com a fixação mediante sutura ${ }^{(4,12,17-18)}$

Sabemos que a avaliação do desconforto ocular por meio do questionário de escala análoga não é isenta de viés, principalmente pela subjetividade dos sintomas, com diferenças pessoais no julgamento de diferentes estímulos; entretanto, em nosso estudo, todos os pacientes receberam as mesmas orientações pré e pós-operatórias, dadas pelo mesmo médico, sem distinção entre os diferentes tipos de tratamento. O menor escore da Escala Análoga Visual (EAV) encontrado no grupo 1, traduz o menor desconforto ocular encontrado em todos os períodos estudados. Resultados similares foram encontrado em outros estudos ${ }^{(1,4,19)}$.
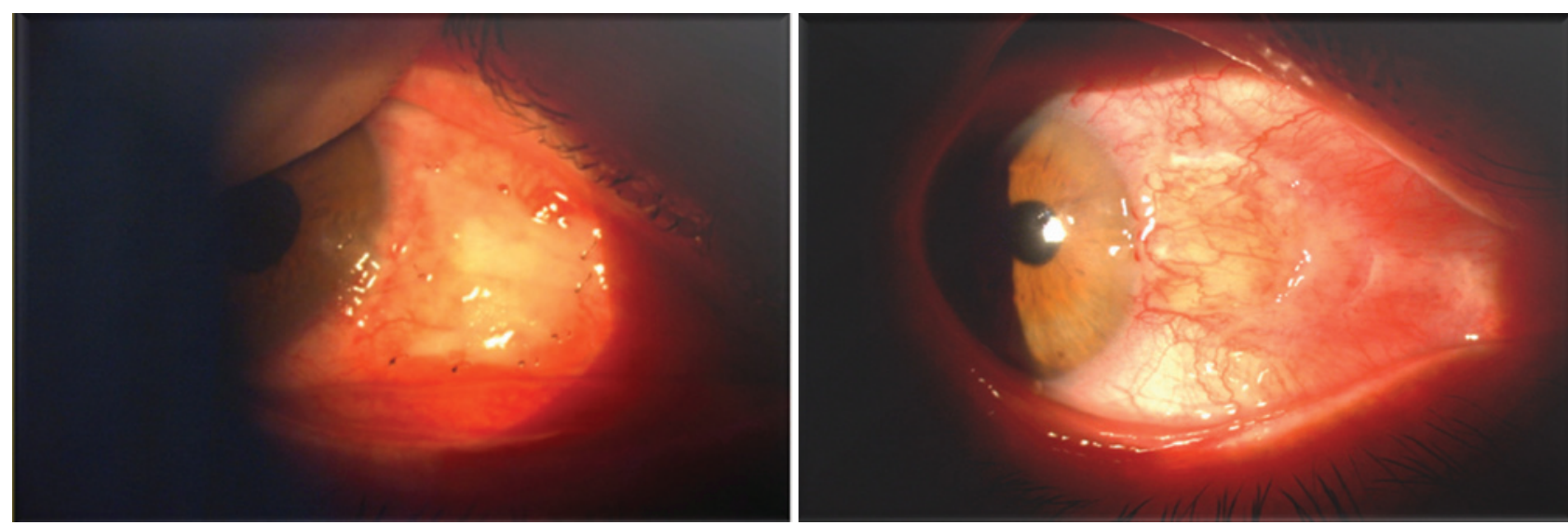

Figura 1. Aspecto do $1^{\circ}$ dia após a cirurgia de exérese de pterígio primário com a fixação do enxerto conjuntival, respectivamente com sutura de Mononylon ${ }^{\circledR}$ e adesivo tecidual de fibrina.

Fonte: Ambulatório de Córnea e Doenças Externas do Departamento de Oftalmologia da Santa Casa de São Paulo. 
Acreditamos que, conjuntamente à EAV, a hiperemia ocular menos intensa apresentada em todas as fases avaliadas neste estudo com a utilização da cola, deveu-se a uma série de fatores: menor manipulação operatória decorrente do menor tempo cirúrgico, propriedades hemostáticas inerentes ao adesivo tecidual de fibrina, que levaram a menor sangramento durante a cirurgia e a não exposição a corpo estranho, que é o fio cirúrgico.

Observamos ainda que, no grupo 1, a EAV e a hiperemia ocular logo após a cirurgia foram semelhantes ao $7^{\circ}$, $14^{\circ}$ e $21^{\circ}$ dia $\mathrm{PO}$, em comparação ao grupo 2. Além dos possíveis motivos já mencionados, acreditamos ainda que a retirada do fio cirúrgico no $21^{\circ}$ dia PO tenha aumentado tanto o desconforto quanto a hiperemia ocular nesta data deste grupo.

Enfatize-se que, menor reação inflamatória e desconforto ocular, permitem retorno ao trabalho mais precoce, com restauração do estilo de vida e produtividade.

O índice geral de complicações foi semelhante. Em relação à gravidade das complicações, todas mostraram baixa morbidade, com melhora via tratamento clínico.

Como o presente estudo, estudos anteriores demonstraram taxas de recidivas semelhantes ${ }^{(1,17)}$ e outros menores ${ }^{(4,20-22)}$ com a utilização de adesivo tecidual de fibrina quando comparados com a fixação mediante sutura. Acreditamos que a ausência do fio cirúrgico proporcione menor migração de células inflamatórias, diminuindo o potencial para a ocorrência de recidivas. Porém, ainda são necessários estudos posteriores com grandes amostras e maior tempo de seguimento para chegarmos a um consenso.

\section{CONCLUSÃO}

Adesivo tecidual de fibrina reduziu o tempo cirúrgico, desencadeou menor hiperemia conjuntival e desconforto no período pósoperatório, com índice de recidiva semelhante, em comparação ao uso de sutura com monofilamento de nylon 10.0, demonstrando ser excelente opção para a fixação do enxerto conjuntival para o tratamento cirúrgico de pterígio primário.

\section{REFERÊNCIAS}

1. Bahar I, Weinberger D, Dan G, Avisar R. Pterygium surgery: fibrin glue versus Vicryl sutures for conjunctival closure. Cornea. 2006;25(10):1168-72.

2. Davidoff F, Tarnow EG. Who's the author? Problems with biomedical authorship, and some possible solutions. Report of the Council of Biology Editors (now Council of Science Editors from the Task Force on Authorship, February 2000 [Internet]. New York: Council of Science Editors; 2000. [cited 2010 Nov21]. Available from: http://www.councilscienceeditors.org/ files/public/V23n4p111-119.pdf
3. Ferraz FH, Schellini SA, Hoyama E, Bernardes SR, Padovani CR. Pterígio e alterações da curvatura corneana. Arq Bras Oftalmol. 2002;65(5):533-6.

4. Hercules LA, Viveiros MM, Schellini SA, Candeias J, Padovani CR. Exposição de fibroblastos da cápsula de Tenon de pterígios à ciclosporina 0,05\%. Arq Bras Oftalmol. 2006:69(6):831-5.

5. Koranyi G, Seregard S, Kopp ED. Cut and paste: a no suture, small incision approach to pterygium surgery. Br J Ophthalmol. 2004;88(7):911-4. Comment in: Br J Ophthalmol. 2005; 89(3):392; author reply 392.

6. Luanratanakorn P, Ratanapakorn T, Suwan-apichon O, Chuck RS. Randomised controlled study of conjuctival autograft versus amniotic membrane graft in pterygium excision. $\mathrm{Br} J$ Ophthalmol;:2006;90(12):1476-80.

7. Jaros PA, DeLuise VP. Pingueculae and pterygia. Surv Ophthalmol. 1988;33(1):41-9.

8. Schellini SA, Veloso CE, Lopes W, Padovani CR. Características de portadores de pterígio na região de Botucatu. Arq Bras Oftalmol. 2005;68(3):291-4

9. Maheshwari S. Pterygium-induced corneal refractive changes. Indian J Ophthalmol. 2007; 55(5):383-6.

10. Sánchez-Thorin JC, Rocha G, Yelin JB. Meta-analysis on the recurrence rates after bare sclera resection with and without mitomycin C use and conjunctival autograft placement in surgery for primary pterygium. Br J Ophthalmol. 1998;82(6):661-5.

11. Kenyon KR, Wagoner MD, Hettinger ME. Conjunctival autograft transplantation for advanced and recurrent pterygium. Ophthalmology. 1985;92(11):1461-70.

12. Tan DT, Chee SP, Dear KB, Lim AS. Effect of pterygium morphology on pterygium recurrence in a controlled trial comparing conjunctival autografting with bare sclera excision. Arch Ophthalmol. 1997;115(10):1235-40. Erratum in: Arch Ophthalmol. 1998; 116(4):552.

13. Bahar I, Weinberger D, Gaton DD, Avisar R. Fibrin glue versus Vicryl sutures for primary conjunctival closure in pterygium surgery: long-term results. Curr Eye Res. 2007;32(5): 399405.

14. Schwartz M, Madariaga J, Hirose R, Shaver TR, Sher L, Chari R, et al. Comparison of a new fibrin sealant with standard topical hemostatic agents. Arch Surg. 2004;139(11):1148-54.

15. Wang GJ, Hungerford DS, Savory CG, Rosenberg AG, Mont MA, Burks SG, et al. Use of fibrin sealant to reduce bloody drainage and hemoglobin loss after total knee arthroplasty: a brief note on a randomized prospective trial. J Bone Joint Surg Am. 2001; 83-A(10): 1503-5.

16. Prabhasawat $P$, Barton K, Burkett G, Tseng SC. Comparison of conjunctival autografts, amniotic membrane grafts, and primary closure for pterygium excision. Ophthalmology. 1997;104(6):974-85.

17. Parell GJ, Becker GD. Comparison of absorbable with nonabsorbable sutures in closure of facial skin wounds. Arch Facial Plast Surg. 2003;5(6):488-90

18. Marticorena J, Rodriguez-Ares MT, Touriño R, Mera P, Valladares M, Martinzes-de-la-Casa $J M$, et al. Pterygium surgery: conjunctival autograft using fibrin adhesive. Cornea. 2006; 25(1):34-6

19. Uy HS, Reyes JM, Flores JD, Lim-Bon-Siong R. Comparison of fibrin glue and sutures for attaching conjunctival autografts after pterygium excision. Ophthalmology. 2005;112(4): 667-71.

20. Ozdamar Y, Mutevelli S, Han U, lleri D, Onal B, llhan O, et al. A comparative study of tissue glue and Vicryl suture for closing limbal-conjunctival autografts and histologic evaluation after pterygium excision. Cornea. 2008;27(5):552-8.

21. Farid M, Pirnazar R. Pterygium recurrence after excision with conjunctival autograft: a comparison of fibrin tissue adhesive to absorbable sutures. Cornea. 2009;28(1):43-5.

22. Karalezli A, Kucukerdonmez C, Akova YA, Altan-Yaycioglu R, Borazan M. Fibrin glue versus sutures for conjunctival autografting in pterygium surgery: a prospective comparative study. Br J Ophthalmol. 2008;92(9):1206-10. 